\title{
Bound states near a moving charge in a quantum plasma
}

\author{
D. Else, ${ }^{1}$ R. Kompaneets, ${ }^{1}$ and S. V. Vladimirov ${ }^{1,2}$ \\ ${ }^{1}$ School of Physics, The University of Sydney, New South Wales 2006, Australia \\ ${ }^{2}$ Joint Institute for High Temperatures, Russian Academy of Sciences, \\ ul. Izhorskaya 13 bld 2, 127412 Moscow, Russia
}

(Dated: September 5, 2018)

\begin{abstract}
It is investigated how the shielding of a moving point charge in a one-component fully degenerate fermion plasma affects the bound states near the charge at velocities smaller than the Fermi one. The shielding is accounted for by using the Lindhard dielectric function, and the resulting potential is substituted into the Schrödinger equation in order to obtain the energy levels. Their number and values are shown to be primarily determined by the value of the charge and the quantum plasma coupling parameter, while the main effect of the motion is to split certain energy levels. This provides a link between quantum plasma theory and possible measurements of spectra of ions passing through solids.
\end{abstract}

\section{INTRODUCTION}

Quantum plasmas have recently received a rapidly growing interest [1-7]. The motivation is related, in particular, to nanostructured metallic and semiconductor materials [7, 8], laser-plasma systems [9, 10], astrophysics [11], and ultracold plasmas [12]. Quantum plasmas can be described by various models including the hydrodynamic one with the Bohm potential [7, 13], kinetic Wigner-Poisson model (a quantum analogue of the classical Vlasov-Poisson system) 7, 13, quantum relativistic plasmadynamics [2], and other models [7, 14]. Based on these and similar approaches, recently there have been studies of quantum effects on various plasma phenomena including two-stream instability [15], ion-acoustic waves [16, 17], spin effects on the plasma dispersion [18, 19], whistlers [20], Bernstein modes [21], Zakharov dynamics [22], and thermodynamic properties [23, 24].

As a part of research in this field, there have been numerous works to link theoretical models to practice. For instance, already in 1956, Watanabe showed that his measurements of energy losses and scattering angles of electrons passing through a thin metallic film are in a remarkable agreement with the quantum plasma dispersion relation that can be derived from the WignerPoisson model [25]. As regards recent works, Manfredi and Hervieux performed quantum plasma simulations that reproduce low frequency nonlinear oscillations revealed in transient reflection experiments on thin films [26]. Furthermore, Marklund et al. demonstrated that the quantum collisionless damping of surface plasmons poses a fundamental size limit for plasmonic devices [27].

Another link between theory and practice is related to spectra of ions moving in quantum plasmas. Indeed, a plasma shields the Coulomb potential of a moving charge and thus affects the bound electron states near it. This was demonstrated experimentally in the regime where the charge velocity exceeds the Fermi one [28, 29]. In this regime, the effect has been studied in a number of theoretical papers [29 34].

The object of this Letter is to investigate this effect for charge velocities smaller than the Fermi one. In this regime, as opposed to that where the charge velocity exceeds the Fermi one, two factors may be particularly significant. The first one is the spread of velocities in a fully degenerate distribution, as the plasma can no longer be considered as cold or almost cold. The second one is the quantum recoil/tunneling. Its role in the shielding of a moving charge was studied in detail in Ref. [35].

\section{MODEL}

We consider a point charge $+Z$ e representing an ion and moving through a plasma at constant velocity $\mathbf{v}$. Here, $e$ is the elementary charge. The plasma is assumed to be a fully degenerate electron gas immersed in a neutralizing background. We adopt the frame of the moving ion and use it as our origin of coordinates.

In describing the quantum-mechanical dynamics of a single electron near the ion in the presence of the plasma, we consider the response of the plasma to be solely due the ion; this is justified when the plasma responds much more strongly to the ion than the electron, as will be the case [34] for $Z \gg 1$. Thus, the problem is reduced to solving the time-independent Schrödinger equation for the one-electron wavefunction $\psi(\mathbf{r})$, namely

$$
-\frac{\hbar^{2}}{2 m_{e}} \nabla^{2} \psi-e \varphi(\mathbf{r}) \psi=E \psi
$$

where $m_{e}$ is the mass of the electron and $\varphi(\mathbf{r})$ is the electrostatic potential generated by the ion in the plasma. Note that, due to the rotational symmetry of the potential about the axis of motion, we can impose the dependence on the azimuthal angle $\phi$ in spherical or cylindrical coordinates to be of the form $\psi \propto e^{i m \phi}$ for some integer value of the magnetic quantum number $m$. The binding energies will be identical for positive and negative $m$.

The potential $\varphi(\mathbf{r})$ can be calculated in the linear ap- 
proximation by:

$$
\begin{aligned}
\varphi(\mathbf{r}) & =\frac{Z e}{4 \pi \epsilon_{0}}\left(\frac{1}{r}\right. \\
& \left.+\frac{1}{2 \pi^{2}} \int \frac{\exp (i \mathbf{k} \cdot \mathbf{r})}{k^{2}}\left[\frac{1}{D(\mathbf{k} \cdot \mathbf{v}, \mathbf{k})}-1\right] d \mathbf{k}\right),
\end{aligned}
$$

where $D(\omega, \mathbf{k})$ is the dielectric response function of the plasma and $\epsilon_{0}$ is the permittivity of free space. Here we use Lindhard's dielectric function [36, 37] for the response of fully degenerate electrons, which reads

$$
\begin{aligned}
& D(\omega, \mathbf{k})=1+ \\
& \frac{3 \omega_{p}^{2}}{k^{2} v_{F}^{2}} \frac{F(\omega+i \nu+a, k)-F(\omega+i \nu-a, k)}{2 a},
\end{aligned}
$$

where

$$
\begin{gathered}
a=\frac{\hbar k^{2}}{2 m_{e}} \\
F(\Omega, k)=\frac{\Omega}{2}+\frac{\left(k v_{F}\right)^{2}-\Omega^{2}}{4 k v_{F}} \ln \left(\frac{\Omega+k v_{F}}{\Omega-k v_{F}}\right),
\end{gathered}
$$

$v_{F}=\left(3 \pi^{2}\right)^{1 / 3} \hbar n_{e}^{1 / 3} / m_{e}$ is the electron Fermi velocity, $\omega_{p}=\sqrt{n_{e} e^{2} /\left(m_{e} \epsilon_{0}\right)}$ is the electron plasma frequency, and $n_{e}$ is the electron number density; $\nu$ represents an infinitesimal positive number. The Lindhard response function can be derived from the Wigner-Poisson system and includes both the quantum tunneling and the degeneracy. The potential resulting from the Lindhard dielectric function has been studied in Refs. [31, 33, 35]. The applicability of our model is analyzed in the discussion section.

We have solved Eq. (1) numerically by finite-difference discretization, with the resulting sparse matrix eigenvalue problem solved using the ARPACK library [38]. The potential $\varphi(\mathbf{r})$ is found by numerical integration of Eq. (2). The bound states in this problem are determined (up to scaling) by the following dimensionless parameters: $Z$, the atomic number of the ion; the ratio $v / v_{F}$; and $H=\hbar \omega_{p} /\left(m_{e} v_{F}^{2}\right)$, which characterizes the strength of the quantum recoil and is also related to the plasma coupling parameter.

\section{RESULTS}

Note that as $H \rightarrow 0$, the quantum tunneling becomes unimportant to the screening so that the nondimensionalized potential [i.e. with position normalized to $\lambda=v_{F} / \omega_{p}$, and the potential normalized to $\left.Z e /\left(4 \pi \epsilon_{0} \lambda\right)\right]$ tends towards the "semiclassical" form. The "semiclassical" form implies that it can be obtained from the classical Vlasov-Poisson system with the fully degenerate velocity distribution. In this limit, the potential surrounding a stationary test charge tends to the DebyeHückel one, with the resulting screening length being equal to the Thomas-Fermi length $\lambda / \sqrt{3}$.

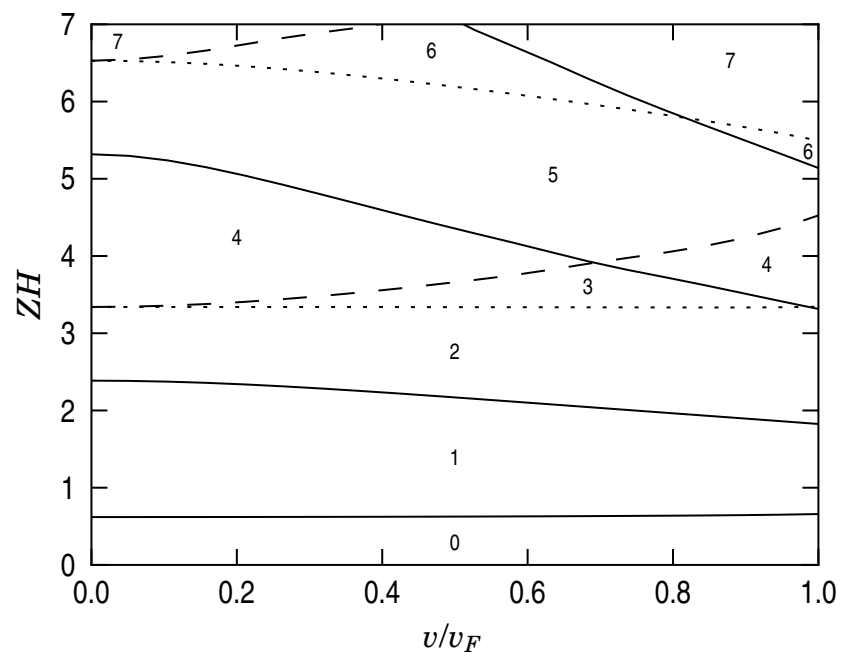

FIG. 1: The number of distinct energy levels (shown by numerals $0,1, \ldots, 7)$ as a function of $v / v_{F}$ (horizontal axis) and $Z H$ (vertical axis), in the limit $H \rightarrow 0, Z H$ held constant. The lines indicate the boundaries between the areas corresponding to different numbers of distinct energy levels. The line style reflects the quantum numbers (with the $l$ quantum number determined by tracing back to $v=0$ ) of the bound state that appears when the line is crossed: solid for $l=0, m=0$; dashed for $l=1, m=0$; dotted for $l=1, m= \pm 1$.

Once the non-dimensionalized potential has been specified, the bound states depend (up to scaling) only on $Z H$. The physical meaning of the product $Z H$ is that it is equal (up to a constant coefficient of the order of unity) to $\lambda /\left(a_{0} / Z\right)$, where $a_{0}=4 \pi \epsilon_{0} \hbar^{2} /\left(m_{e} e^{2}\right)$ is the Bohr radius. Note that $a_{0} / Z$ is the characteristic scale of the ground state of the ion in vacuum. Therefore it is informative to consider the limit $H \rightarrow 0, Z H$ held constant.

Fig. 1 shows the number of energy levels in the limit $H \rightarrow 0, Z H$ held constant. As $Z H$ increases, the ratio of the screening length to the dimensions of individual bound states becomes larger, and thus more bound states appear and the binding energy of levels increases, tending to the bare Coulomb value $|E|=0.5 Z / n^{2}$ a.u. (where $n$ is the principal quantum number) as $Z H \rightarrow \infty$. The splitting of energy levels occurs because the isotropy of the potential is broken. Fig. 2 shows how a state which at $v=0$ is isotropic is distorted by the changes to the potential.

In general, the effect of the motion on the potential is greater screening (and the development of a repulsive potential) behind the moving ion (and to a lesser extent in the direction perpendicular to the motion), and decreased screening in front of the ion [35]. Thus, since states with different angular quantum numbers (i.e. $l$ and $m$ ) have different angular dependencies, they are affected by the anisotropy of the potential in different ways, as can be seen in Fig. 1, In particular, some of the boundary curves in Fig. 1 cross, with the result that for particular values 
a)

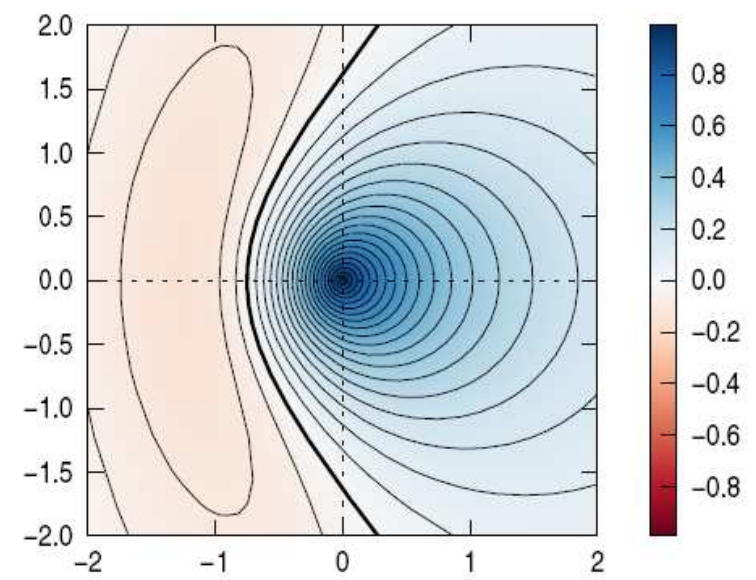

b)

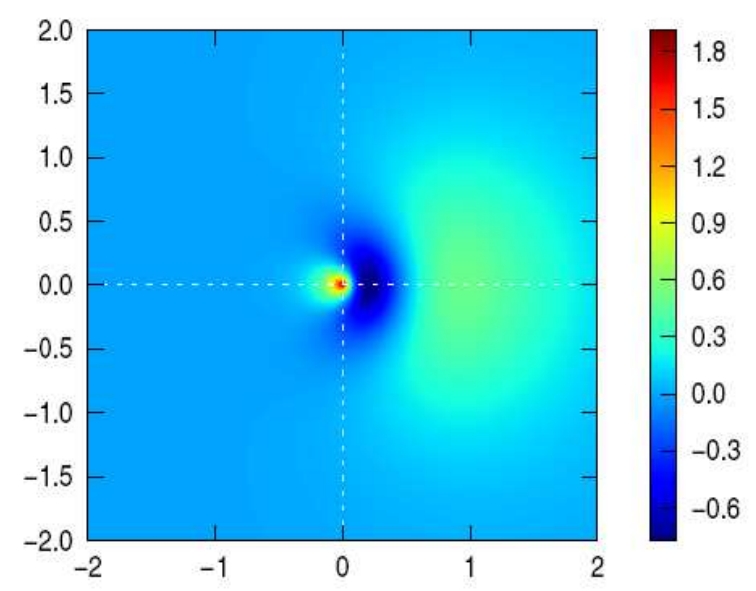

FIG. 2: The potential generated by the ion, at $v=0.9 v_{F}$ in the limit $H \rightarrow 0$; and one of the bound states in this potential, with $Z H$ held constant and equal to 6 . The ion is located at the origin and moving to the right; the coordinates are in units of $\lambda=v_{F} / \omega_{p}$. (a) The ratio of the potential $\varphi(\mathbf{r})$ to the unscreened Coulomb potential $Z e /\left(4 \pi \epsilon_{0} r\right)$. The contours are equally spaced, and the thicker contour corresponds to the boundary between positive and negative potential. (b) The wavefunction $\psi(\mathbf{r})$ for a particular bound state, which can be continuously traced back to the bound state near a stationary test charge with quantum numbers $n=3, l=m=0$.

of $Z H$, there can be narrow ranges of $v / v_{F}$ in which a bound energy level disappears, or an additional level appears. However, as states become more tightly bound, their spatial extent becomes much smaller than $\lambda$, and they cease to be greatly affected by the motion.

Interestingly, the minimal value of $Z H$ at which at least one bound state exists is almost independent on $v / v_{F}$ and is about 0.6. At the same time, the value of $Z H$ at which the second level appears shows a more noticeable dependence on velocity. This value decreases from about 2.3 at zero velocity to about 1.8 at the Fermi velocity and thus changes by about $20 \%$.

In the fully quantum case of finite $H$ (but less than

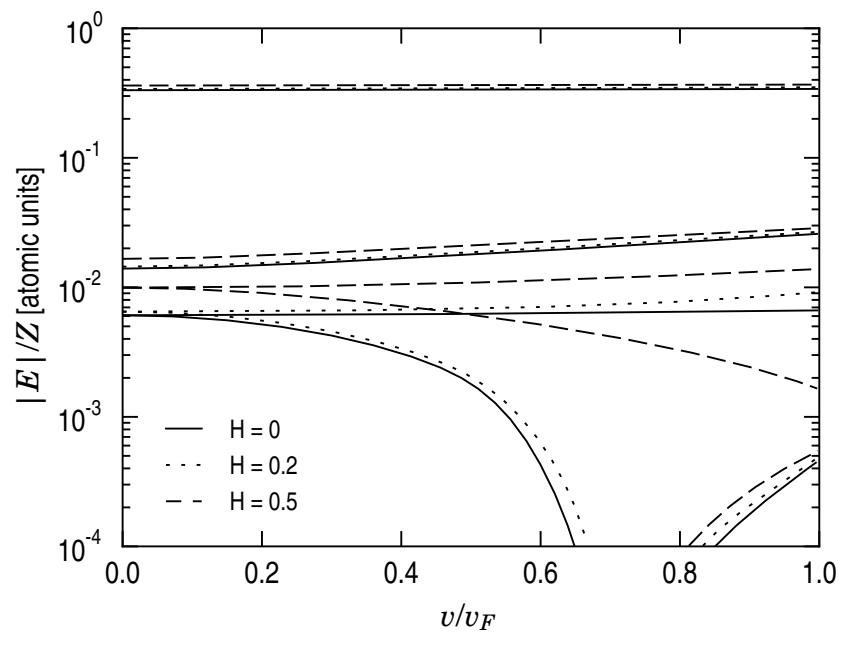

FIG. 3: The binding energies (divided by the atomic number $Z$ ) as a function of velocity for different values of $H$, but all with $Z H \approx 3.82$. Different lines of the same style correspond to different bound states for the same parameters; all possible bound states are shown for given parameters.

unity), the quantum tunneling does not introduce qualitative changes to the above, though it can make noticeable quantitative changes. This can be seen in Fig. 3. It shows that for a fixed $Z H$ the binding energies normalized by $Z$ increase with $H$ and tend to their semiclassical values at $H \rightarrow 0$. Thus, the main effect of the quantum tunneling on Fig. 1 would be to displace the boundary curves down.

\section{DISCUSSION}

The use of our model is limited because it does not include correlations of plasma electrons, nonlinear and relativistic plasma effects as well as the plasma response to the bound electron. Let us discuss the corresponding applicability limits in terms of the parameters $H$ and $Z$.

The neglect of correlations of plasma electrons is justified when $H \ll 1$. Indeed, the use of the collisionless mean-field approximation requires that the quantum plasma coupling parameter is small. The latter is defined as $\Gamma=\left[e^{2} n^{1 / 3} /\left(4 \pi \epsilon_{0}\right)\right] /\left[m_{e} v_{F}^{2} / 2\right]$ which is the ratio of the characteristic potential energy of interaction between neighboring electrons to the Fermi energy. The parameter $\Gamma$ can be expressed as $\Gamma=H^{2}\left(3 \pi^{2}\right)^{2 / 3} /(2 \pi)$, so we get the requirement $H \ll 1$. In the limit $H \rightarrow 0$, however, the quantum tunneling disappears [13]. Thus, the fact that our model includes the quantum tunneling but does not include particle correlations makes it inconsistent in a certain sense. Nevertheless, it is widely used in the literature, i.e. at small $H$ the quantum tunneling is believed to be more important than particle correlations.

The use of the linear response formalism [Eq. (2)] requires $H \ll 1 / Z^{1 / 3}$. This limitation can be derived from the condition that the potential energy of the interaction 
of a plasma electron with the test charge at the length $\lambda$ is much smaller than the Fermi energy.

The relativistic plasma effects are negligible when $H \gg 0.06$. This condition follows from the requirement that the Fermi velocity is much smaller than the speed of light. The latter requirement can be written as $H \gg 2 \sqrt{\alpha /(3 \pi)} \approx 0.06$, where $\alpha=e^{2} /\left(4 \pi \epsilon_{0} \hbar c\right) \approx 1 / 137$ is the fine structure constant.

The plasma response to the bound electron should be unimportant when $Z \gg 1$. In this case the ion should generate a significantly stronger plasma response due to its larger charge. The requirement $Z \gg 1$ is also evident from the results of Ref. 34].

The four above requirements can be easily satisfied at small $H$ and large $Z$. Note that the relativistic restriction is formally not met in the limit $H \rightarrow 0, Z H$ held constant. This means that taking this limit within our model gives results that apply at not extremely small, though sufficiently small, values of $H$.

It follows that metals are at the edge of applicability of our model. For instance, for aluminum the parameter $H$ is about 0.7 , as can be deduced from its Fermi energy, $11.7 \mathrm{eV}$. This value of $H$ suggests that nonideal plasma effects [3, 9] can provide a non-negligible contribution. Nevertheless, the Lindhard dielectric function can apply quite well in metals, as evidenced by the experiment of Watanabe [25].

\section{CONCLUSION}

We have studied how the shielding a moving charge in a fully degenerate electron gas affects the bound elec- tron states near the charge for velocities smaller than the Fermi velocity. We accounted for the shielding by using the Lindhard dielectric function.

The main result is that the number of distinct energy levels is primarily determined by the parameter $Z H$, while the main effect of the motion is to split certain energy levels. In the semiclassical limit, i.e. $H \rightarrow 0, Z H$ held constant, the minimal value of $Z H$ at which at least one bound state exists is almost independent of velocity and is about 0.6, while the value of $Z H$ at which the second level appears varies from 2.3 to 1.8 with velocity. In the fully quantum case of finite $H$ (but less than unity), the quantum tunneling does not introduce qualitative changes, but it can introduce noticeable quantitative changes. They are that the binding energies normalized by $Z$ increase with $H$, for a fixed $Z H$.

This provides a link between quantum plasma theory and possible measurements of spectra of ions passing trough solids. For instance, the measured positions of spectral lines as well as their splitting could be compared to theoretical values. This might allow, in particular, assessing the role of the effects not included in the linear mean-field model, for various conditions.

\section{Acknowledgments}

The work was partially supported by the Australian Research Council. R.K. acknowledges the receipt of a Professor Harry Messel Research Fellowship funded by the Science Foundation for Physics within the University of Sydney.
[1] G. Manfredi, Fields Inst. Commun. 46, 263 (2005).

[2] D. B. Melrose, Quantum Plasmadynamics: Unmagnetized Plasmas (Springer, New York, 2008).

[3] V. E. Fortov, Extreme states of matter on Earth and in the Cosmos (Springer, Berlin Heidelberg, 2008).

[4] P. K. Shukla, Nature Phys. 5 (92) (2009).

[5] P. K. Shukla and B. Eliasson, Plasma Phys. Control. Fusion 52, 124040 (2010).

[6] P. K. Shukla and B. Eliasson, Phys. Usp. 53, 51 (2010).

[7] G. Manfredi, P.-A. Hervieux, Y. Yin and N. Crouseilles, Atomic-Scale Modeling of Nanosystems and Nanostructured Materials (Springer, Berlin Heidelberg, 2010), pp. $1-44$.

[8] G. Manfredi and P.-A. Hervieux, Appl. Phys. Lett. 91, 061108 (2007).

[9] V. E. Fortov, A. G. Khrapak, and I. T. Yakubov, Physics of Strongly Coupled Plasmas (Oxford University Press, New York, 2005).

[10] M. Marklund and P. K. Shukla, Rev. Mod. Phys. 78, 591 (2006).

[11] G. Brodin, M. Marklund, B. Eliasson and P. K. Shukla, Phys. Rev. Lett. 98, 125001 (2007).

[12] W. Li, P. J. Tanner and T. F. Gallagher, Phys. Rev. Lett.
94, 173001 (2005).

[13] G. Manfredi and F. Haas, Phys. Rev. B 64, 075316 (2001).

[14] D. Kremp, M. Schlanges and W.-D. Kraeft, Quantum Statistics of Nonideal Plasmas (Springer, Berlin, 2005).

[15] F. Haas, A. Bret and P. K. Shukla, Phys. Rev. E 80, 066407 (2009).

[16] S. Ali, W. M. Moslem, P. K. Shukla and R. Schlickeiser, Phys. Plasmas 14, 082307 (2007).

[17] A. Mushtaq and D. B. Melrose, Phys. Plasmas 16, 102110 (2009).

[18] M. Marklund and G. Brodin, Phys. Rev. Lett. 98, 025001 (2007).

[19] G. Brodin, M. Marklund, J. Zamanian, A. Ericsson and P. L. Mana, Phys. Rev. Lett. 101, 245002 (2008).

[20] A. P. Misra, G. Brodin, M. Marklund and P. K. Shukla, Phys. Rev. E 82, 056406 (2010).

[21] B. Eliasson and P. K. Shukla, Phys. Plasmas 15, 102102 (2008).

[22] M. Marklund, Phys. Plasmas 12, 082110 (2005).

[23] V. S. Filinov, M. Bonitz, V. E. Fortov, W. Ebeling, H. Fehske, D. Kremp, W. D. Kraeft, V. Bezkrovniy and P. Levashov, J. Phys. A: Math. Gen. 39, 4421 (2006). 
[24] V. S. Filinov, P. R. Levashov, A. V. Botan, M. Bonitz and V. E. Fortov, J. Phys. A: Math. Theor. 42, 214002 (2009).

[25] H. Watanabe, J. Phys. Soc. Japan 11, 112 (1956).

[26] G. Manfredi and P.-A. Hervieux, Phys. Rev. B 72, 155421 (2005).

[27] M. Marklund, G. Brodin, L. Stenflo and C. S. Liu, Europhys. Lett. 84, 17006 (2008).

[28] F. Bell, H.-D. Betz, H. Panke and W. Stehling, J. Phys. B: Atom. Molec. Phys. 9, L443 (1976).

[29] D. H. Jakubassa, J. Phys. C: Solid State Phys. 10, 4491 (1977).

[30] V. N. Neelavathi, R. H. Ritchie and W. Brandt, Phys. Rev. Lett. 33, 302 (1974).

[31] M. H. Day, Phys. Rev. B 12, 514 (1975).
[32] P. M. Echenique and R. H. Ritchie, Phys. Rev. B 21, 5854 (1980).

[33] A. Mazarro, P. M. Echenique and R. H. Ritchie, Phys. Rev. B 27, 4117 (1983)

[34] P. M. Echenique, W. Brandt and R. H. Ritchie, Phys. Rev. B 33, 43 (1986).

[35] D. Else, R. Kompaneets and S. V. Vladimirov, Phys. Rev. E 82, 026410 (2010).

[36] J. Lindhard, K. Dan. Vidensk. Selsk. Mat. Fys. Medd. 28, 1 (1954).

[37] E. M. Lifshitz and L. P. Pitaevskii, Physical Kinetics (Pergamon, Oxford, 1981), pp. 161-167.

[38] R. B. Lehoucq, D. C. Sorensen and C. Yang, ARPACK Users' Guide (SIAM, Philadephia, 1998). 